\title{
Taux d'occupation des médecins en Suisse
}

\author{
Voilà plusieurs décennies que la FMH tient une statistique médicale. Ces données \\ sont utiles aux sociétés de discipline médicale, aux sociétés cantonales de méde- \\ cine, aux médecins ainsi qu'à différentes organisations pour les prises de position \\ politiques, les argumentaires, la planification des besoins et les enquêtes. Le dépar- \\ tement Données, démographie et qualité (DDQ) publie régulièrement des articles à \\ ce sujet dans le Bulletin des médecins suisses.
}

\begin{abstract}
Stefanie Hostettler ${ }^{\text {, }}$ Renato Laffranchi ${ }^{b}$, Esther Kraftc
\end{abstract}

a Dr sc., EPF Zurich, département Données, démographie et qualité (DDQ)

b Dr, General Manager NewIndex

c lic. rer. oec., responsable du département Données, démographie et qualité (DDQ) de la FMH
Correspondance: FMH/Département DDQ Elfenstrasse 18 CH-3000 Berne 15 Tél. 0313591111 ddq[at]fmh.ch

\section{Introduction}

Depuis la révision de la statistique médicale de la FMH en 2008, les médecins peuvent déclarer, actualiser et vérifier les données concernant leur activité professionnelle, et désormais aussi leur taux d'occupation, sur le portail des membres www.myfmh.ch. De 2008 à 2012, le taux de participation n'a cessé d'augmenter et atteint désormais plus de 5500 médecins. Grâce à cette base de données importante et fiable, la FMH dispose d'arguments solides à faire valoir dans les discussions de politique de santé et peut ainsi défendre au mieux les intérêts des médecins. Les évaluations relatives aux volumes de travail des médecins en fonction du taux d'occupation revêtent beaucoup d'importance notamment dans le but de prévoir les futurs modèles de soins.

Le présent rapport entend clarifier les questions ayant trait au taux d'occupation des médecins et livrer des faits. Il a pour but de montrer le taux d'occupation moyen des médecins et son évolution au cours des cinq dernières années tout en établissant des parallèles entre taux d'occupation, sexe, discipline médicale ou situation géographique par exemple.

La FMH remercie chaleureusement les médecins pour leur collaboration; sans eux, de telles analyses ne seraient tout simplement pas possibles. Elle invite ceux qui n'ont pas encore participé à le faire, car ils contribuent ainsi à l'établissement d'une statistique médicale solide et fiable.

\section{Données utilisées}

Les données de la statistique médicale servent à analyser le taux d'occupation des médecins. Les données disponibles concernent les années 2008 à 2012 et se basent sur les auto-déclarations des médecins. Afin d'analyser et d'évaluer la fiabilité des données de la $\mathrm{FMH}$, les indications concernant le taux d'occupation ont été comparées à celles de la facturation. La pertinence de l'échantillon obtenu a ensuite été contrôlée.

\section{Plausibilisation}

Afin que les évaluations et les déclarations présentées ici reposent sur un socle de données solide, la FMH a, grâce au soutien de NewIndex (www.newindex.ch), vérifié la plausibilité des données obtenues par autodéclaration. Ces données, fournies par 700 médecins libres praticiens, ont ensuite été harmonisées et comparées avec les prestations facturées dans le TARMED et les minutages correspondants. Toutes ces évaluations ont eu lieu de manière anonymisée afin qu'on ne puisse pas remonter à une personne particulière. Etant donné que les médecins ne peuvent pas facturer toutes leurs activités au moyen du TARMED, les minutages ont été rehaussés de 10\% [1] au moment de traiter les données (ce qui correspond à un demi-jour de travail pour un poste à plein temps). Le taux d'occupation moyen par semaine en fonction des minutages et des données auto-déclarées est respectivement de 34,5 et de 35,5 heures de travail hebdomadaires et permet un bon recoupement des deux sources de données.

\section{Taille de l'échantillon}

La taille de l'échantillon $(\mathrm{n}=5567)$ permet d'étendre les résultats de l'évaluation à l'ensemble du corps médical, dans la mesure où aucun biais statistique ne résulte de la sélection de certains groupes de médecins. Les indications quant à une sous- ou sur-représentation de certaines caractéristiques dans l'échantillon (ou dans les groupes d'échantillons) en comparaison avec l'ensemble du corps médical ont été vérifiées selon le sexe, le secteur d'activité et les disciplines médicales. Dans l'ensemble, on peut dire que la fréquence des facteurs susmentionnés est comparable à celle de la population globale. Là où les écarts entre l'échantillon et l'ensemble de la population est

Le département $\mathrm{DDQ}$ se tient à votre disposition pour de plus amples informations et des évaluations approfondies: ddq[at]fmh.ch / 0313591111. 
supérieur à 10\%, une pondération a été effectuée (secteurs/femmes dans le domaine hospitalier). Toutes les évaluations ont été effectuées sur la base d'un échantillon d'au moins $\mathrm{n}=30$.

Les calculs portant sur les échantillons du secteur ambulatoire $n=3758$ et hospitalier $n=1670$ et sur l'ensemble des évaluations ont donné une erreur aléatoire (avec un taux de fiabilité de 99\%: $\mathrm{t}=2,58$, pour une valeur attestée de $50 \%$ ) de $+/-2 \%$ pour le secteur ambulatoire et de $+/-3 \%$ pour le secteur hospitalier. L'analyse effectuée montre que le contenu et les caractéristiques des données de la FMH permettent de tirer des conclusions solides et fiables applicables à l'ensemble de la population médicale.

\section{Taux d'occupation des médecins en exercice}

\section{Quel est le taux d'occupation moyen des médecins en exercice?}

En 2012, les médecins ont travaillé en moyenne 8,8 demi-journées ${ }^{1}$ (tabl. 1) par semaine. Dans le secteur ambulatoire, le taux d'occupation moyen était de 8,3 demi-journées, autrement dit, un demi-jour de moins que dans le secteur hospitalier (9,7 demi-journées). Le taux d'occupation moyen des femmes médecins (secteur ambulatoire: 6,9; hospitalier: 8,9) est inférieur à celui de leurs collègues masculins (secteur ambulatoire: 8,9; hospitalier: 10,1). Ce modèle de répartition n'a guère changé au cours des cinq dernières années.

Alors que dans le secteur hospitalier, la majorité des médecins (77\%) travaille à temps plein, ce taux s'élève à 58\%, à savoir 20\% de moins, dans le secteur ambulatoire. La figure 1 montre que cet écart entre les deux secteurs est principalement lié au sexe. Alors que $32 \%$ des hommes travaillent à temps partiel dans le secteur ambulatoire, ce chiffre atteint $71 \%$ chez les femmes médecins. Celles-ci optent principalement pour des taux d'occupation allant de $60 \%$ à $80 \%$. Dans le domaine hospitalier, la majorité des médecins femmes $(51 \%)$ et hommes $(78 \%)$ travaillent à plein temps. Cela est probablement dû au fait que la formation postgraduée des médecins se déroule principalement dans le secteur hospitalier et que durant cette étape de leur carrière, les médecins ont plus de difficultés à réduire leur temps de travail.

\section{Taux d'occupation et fonction}

Une évaluation selon la fonction confirme l'hypothèse que peu de médecins travaillent à temps partiel au cours de leur formation postgraduée (tabl. 2) et que durant cette phase de leur carrière, la différence entre les sexes est minime. En revanche, au niveau des chefs de clinique et des médecins adjoints le taux d'occupation est plus bas chez les femmes. Ces résultats laissent supposer que durant cette étape de leur carrière, les femmes réduisent leur temps de travail notamment pour des raisons familiales. Bien que depuis de nombreuses années, plus de femmes que d'hommes terminent leurs études de médecine (2012: 55,6\%), le nombre de femmes médecins dans les hôpitaux diminue plus le niveau hiérarchique est élevé. En effet, seuls 9\% de femmes occupent par ex. un poste de médecin-chef. Cet écart est probablement

$\begin{aligned} & \text { Tableau } 1 \\
& \text { Evolution du taux d'occupation de } 2008 \text { à } 2012 .\end{aligned}$
\begin{tabular}{llllll} 
& & & & \\
& $\mathbf{2 0 0 8}$ & $\mathbf{2 0 0 9}$ & $\mathbf{2 0 1 0}$ & $\mathbf{2 0 1 1}$ & $\mathbf{2 0 1 2}$ \\
& $\mathrm{n}=1581$ & $\mathrm{n}=3009$ & $\mathrm{n}=3902$ & $\mathrm{n}=4753$ & $\mathrm{n}=5567$ \\
\hline Secteur ambulatoire & 8,7 & 8,6 & 8,4 & 8,4 & 8,3 \\
\hline Secteur hospitalier & 9,9 & 9,8 & 9,8 & 9,7 & 9,7 \\
\hline Total & $\mathbf{9 , 0}$ & $\mathbf{8 , 9}$ & $\mathbf{8 , 9}$ & $\mathbf{8 , 8}$ & $\mathbf{8 , 8}$ \\
& & & & &
\end{tabular}

Une demi-journée correspond à un volume de travail de 4 à 6 heures.

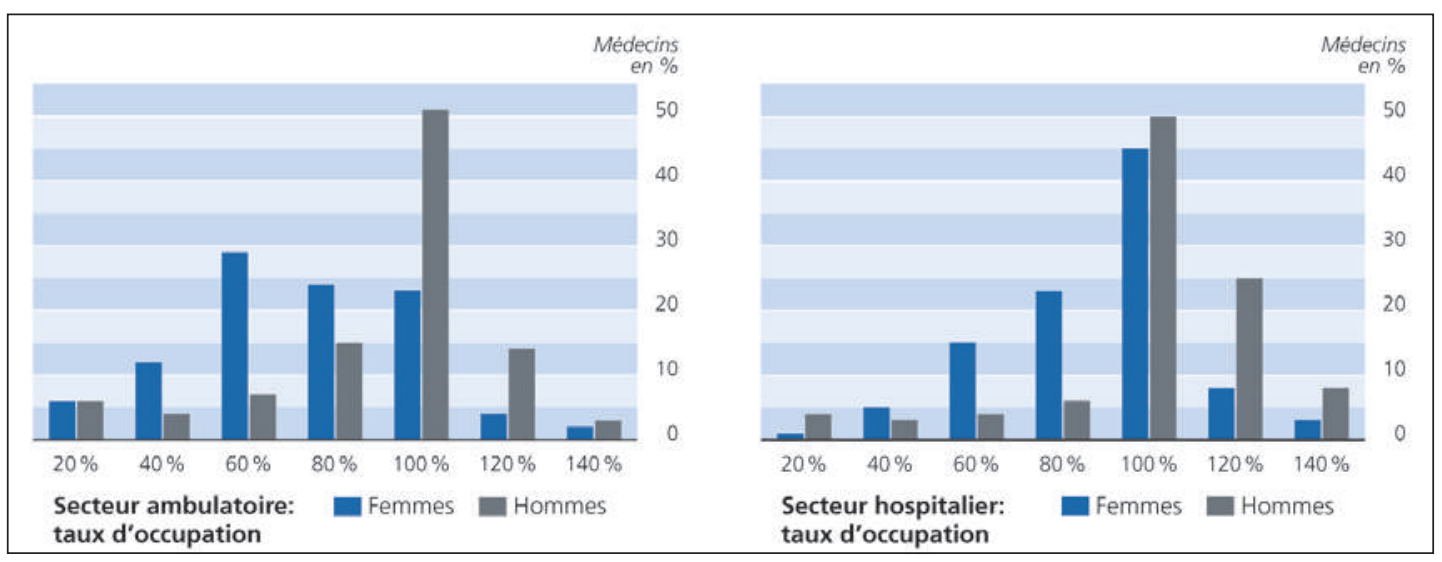

Figure 1

Taux d'occupation en fonction du sexe et du secteur en $2012(n=5428)$. 
dû à la difficulté de concilier vie professionnelle et vie familiale à ce niveau de responsabilité. Afin de limiter la perte de personnel qualifié et de maintenir les opportunités de carrière pour les femmes malgré les impératifs familiaux, il est nécessaire de mettre en place des modèles flexibles de temps de travail dans les hôpitaux, et ce indépendamment du niveau hiérarchique $[2,3]$.

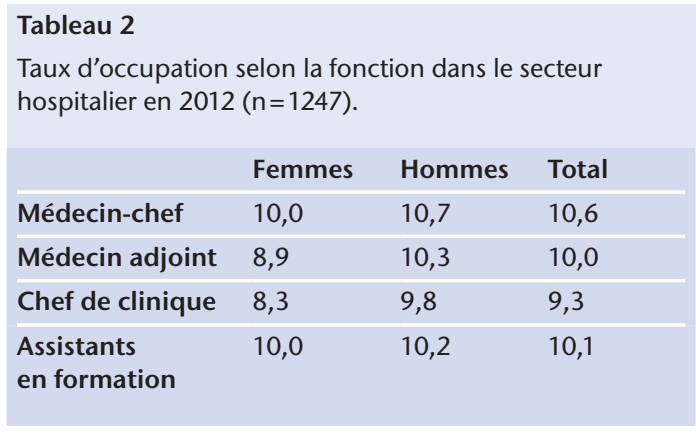

\section{Temps de travail inversement proportionnel} à la taille du cabinet médical

Les chiffres du tableau 3 montrent que le taux d'occupation varie en fonction de la taille du cabinet, c'està-dire que plus le cabinet est grand, plus le taux d'occupation diminue. Il est par ailleurs intéressant de noter qu'il est tout à fait possible de diriger un cabinet individuel à temps partiel (7,5 demi-journées) à l'exemple des femmes dans le tableau ci-dessous.

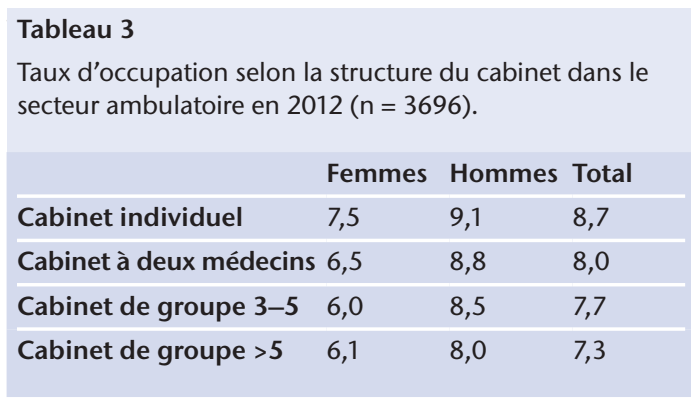

\section{Taux d'occupation et discipline médicale}

Le taux d'occupation à temps partiel est - toutes disciplines confondues - plus fréquent dans le secteur ambulatoire et chez les femmes médecins que dans le secteur hospitalier et chez les hommes. Avec 0,7 demi-journée (secteur ambulatoire) et 1,1 demijournée (secteur hospitalier), les différences sont faibles entre les disciplines, qu'elles soient exercées dans l'un ou l'autre secteur. Les résultats de l'analyse montrent que le travail à temps partiel est particulièrement prisé en psychiatrie probablement en raison du nombre relativement élevé de femmes travaillant en psychiatrie et psychothérapie ainsi qu'en psychiatrie et psychothérapie d'enfants et d'adolescents $(41 \%$ et $63 \%$, contre $15 \%$ en chirurgie par exemple) [4].

\section{C'est en Suisse orientale que le taux d'occupation moyen est le plus élevé}

La figure 2 montre le taux d'occupation des médecins par secteur et par grandes régions. La plupart des grandes régions affichent un taux d'occupation inférieur dans le secteur ambulatoire par rapport au secteur hospitalier. Dans ces deux secteurs, la Suisse orientale caracole en tête en ce qui concerne le taux d'occupation le plus élevé. Comparé à la densité médicale, dans les grandes régions à forte densité médicale (Arc lémanique, Espace Mittelland, Suisse du Nord-Ouest et Zurich) le taux d'occupation moyen est plus bas que dans les grandes régions plutôt rurales de Suisse orientale et centrale ainsi que du Tessin [5]. La comparaison entre cantons universitaires (secteur ambulatoire: 8,1 demi-journées; secteur hospitalier: 9,4 ) versus cantons non universitaires (secteur ambulatoire: 8,4 ; secteur hospitalier: 9,8 ) ne montre pas de différence notoire quant au taux d'occupation.

\section{Conclusions}

Le corps médical suisse se trouve en pleine mutation (pénurie de médecins, répartition des sexes, etc.). Dans ce contexte, il est important d'analyser et de documenter non seulement le développement démographique, mais aussi l'évolution du taux d'occupation des médecins. Les enquêtes effectuées ont en effet montré que de plus en plus de médecins (hommes et femmes) souhaitent travailler à temps partiel $[3,6]$. Afin de rendre cela possible, il convient de créer les conditions nécessaires à la promotion de
2 La répartition par discipline figure dans l'annexe.

\section{Tableau 4}

Taux d'occupation par discipline ${ }^{2}$ en $2012(n=5428)$.

\begin{tabular}{|c|c|c|c|c|c|c|}
\hline & \multicolumn{3}{|c|}{ Ambulatoire } & \multicolumn{3}{|c|}{ Hospitalier } \\
\hline & Femmes & Hommes & Total & Femmes & Hommes & Total \\
\hline Médecins de premier recours & 6,7 & 9,2 & 8,5 & 8,7 & 9,9 & 9,5 \\
\hline Gynécologie et obstétrique & 7,2 & 8,8 & 7,9 & 8,3 & 10,1 & 9,2 \\
\hline Psychiatrie & 7,0 & 8,3 & 7,8 & 7,8 & 9,4 & 8,9 \\
\hline $\begin{array}{l}\text { Discipline spécialisée } \\
\text { sans activité chirurgicale }\end{array}$ & 7,6 & 8,7 & 8,5 & 9,0 & 10,1 & 9,9 \\
\hline $\begin{array}{l}\text { Discipline spécialisée } \\
\text { avec activité chirurgicale }\end{array}$ & 7,0 & 8,4 & 8,1 & 8,7 & 10,3 & 10,0 \\
\hline Autres disciplines & 7,1 & 8,7 & 8,2 & 9,1 & 9,9 & 9,7 \\
\hline
\end{tabular}



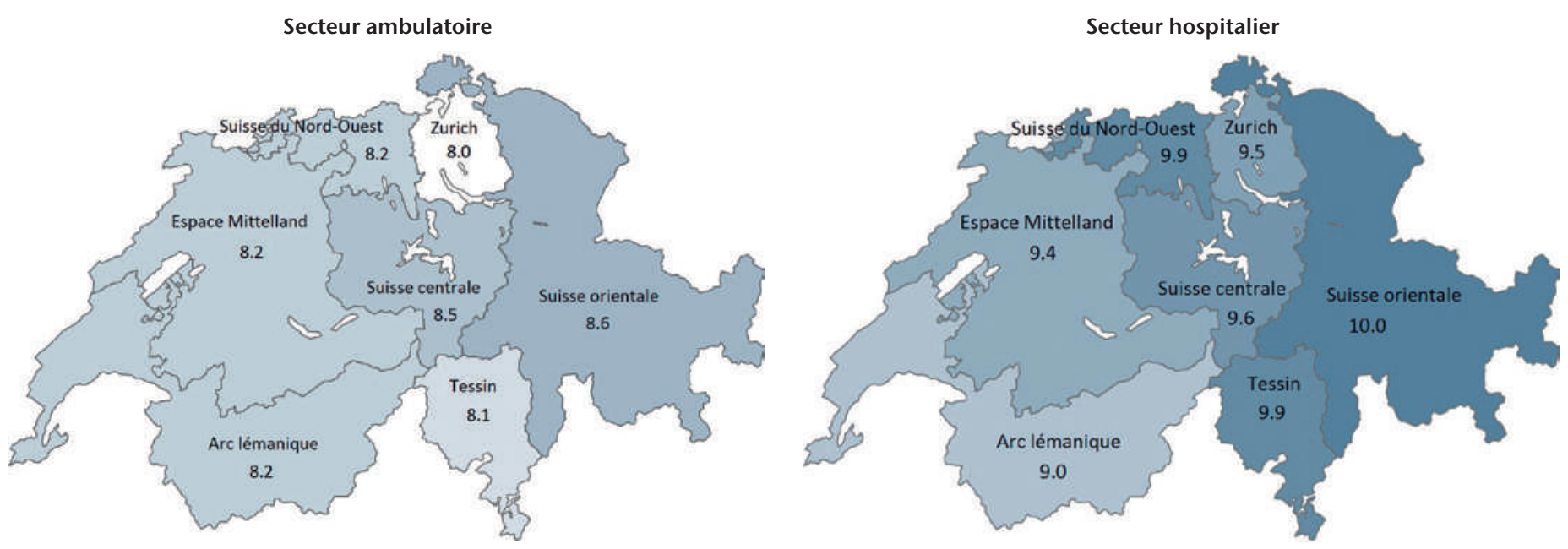

Figure 2

Taux d'occupation par grandes régions en $2012^{3}(n=5428)$.

modèles de travail à temps partiel, qui auraient non seulement des répercussions positives sur la satisfaction au travail mais indirectement aussi sur la qualité des traitements [7]. De plus, il ne faut pas oublier que le travail à temps partiel aggrave la pénurie de médecins puisqu'il faut plus de médecins pour un équivalent plein temps, ce qui rend encore plus urgente l'exigence d'augmenter le nombre de places de formation pré- et postgraduée et de proposer des modèles de travail qui tiennent compte des nouveaux projets de vie. En effet, ce n'est qu'ainsi qu'il sera possible de faire bénéficier le plus largement possible les patients des moyens investis dans la formation. Pour garantir que le système de santé helvétique puisse continuer à fonctionner, la recherche de solutions devra de plus en plus se discuter au niveau politique.

Le présent rapport donne un aperçu du taux d'occupation et pourra servir de base à d'autres études qui permettront une analyse détaillée et de mettre en lumière d'importants aspects liés au taux d'occupation. Par ailleurs, la saisie des heures de travail accomplies en fonction du type d'activité, l'offre et la demande de postes à temps partiel ou les effets du travail à temps partiel sur la structure et l'organisation d'un cabinet médical permettraient de réunir des informations importantes. La FMH a demandé aux organisations qui lui sont affiliées quels étaient les

\section{La statistique médicale: une contribution simple aux effets multiples}

Quel est le taux d'occupation des médecins en exercice? Le travail à temps partiel a-t-il augmenté? Si oui, dans quelles disciplines? Existe-t-il des différences quant à l'âge et au sexe? De telles évaluations sont précieuses pour anticiper les défis et les changements futurs dans le domaine de la santé.

Pour que la statistique médicale puisse à nouveau se fonder sur des données solides et fiables en
2013, nous avons besoin de votre soutien. Nous vous serions reconnaissants de déclarer, actualiser ou contrôler vos données sur le portail des membres myFMH. En agissant ainsi, vous nous aidez à rendre la statistique médicale de la FMH encore plus pertinente!

\section{Enregistrez-vous sur: www.myfmh.ch}

Les détails de la répartition selon les grandes régions figurent dans l'annexe.

\section{Pour anticiper les défis.} La statistique médicale de la FMH.

\section{Déclarez vos données.}

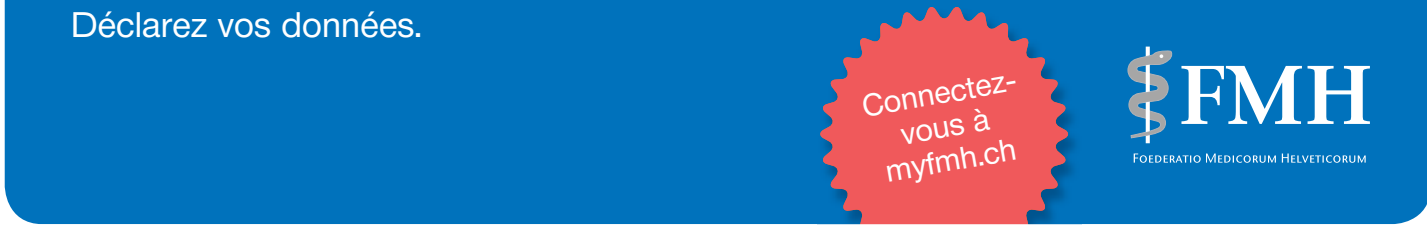


besoins en matière de structure de cabinet et d'offres à temps partiel. Les résultats de cette enquête sont en cours d'évaluation et des idées pour des projets concrets sont actuellement discutées et développées.

\section{Références}

1 Kraft E, Napierala C. Validation des données en vue de la recherche sur la fourniture des soins. Bull Méd Suisses. 2010;91(3):64-6.

2 Kraft E, Hersperger M. Le corps médical en Suisse: la féminisation de la médecine. Bull Méd Suisses. 2009; 90(47):1823-5.

3. www.aargauerzeitung.ch/schweiz/steigenderaerztemangel-junge-mediziner-wollen-nur-nochteilzeit-arbeiten-127271657

4. www.fmh.ch/fr/services/statistique/statistique medicale.html

5. Kraft E, Hersperger M. Le sexe et la région jouent un rôle dans la répartition des médecins. Bull Méd Suisses. 2011;92(48):1854-6.

6. www.beobachter.ch/leben-gesundheit/ medizin-krankheit/artikel/spitalaerzte_arbeitenbis-zum-umfallen/

7. Hostettler S, Hersperger M, Herren D. Le bien-être du médecin influe sur la qualité du traitement. Bull Méd Suisses. 2012;93(18):655-9.

\section{Annexe}

\section{Répartition des disciplines médicales}

Spécialités médicales sans activité chirurgicale: Allergologie/immunologie, angiologie, endocrinologie/diabétologie, gastroentérologie, hématologie, in- fectiologie, cardiologie, oncologie médicale, néphrologie, neurologie, médecine physique et réadaptation, pneumologie, rhumatologie.

Spécialités médicales avec activité chirurgicale: Anesthésiologie, chirurgie, chirurgie de la main, chirurgie cardiaque et vasculaire thoracique, chirurgie pédiatrique, chirurgie maxillo-faciale, neurochirurgie, ophtalmologie, ORL, chirurgie orthopédique, chirurgie plastique, reconstructive et esthétique, urologie.

\section{Autres disciplines:}

Médecine du travail, dermatologie et vénéréologie, médecine intensive, pharmacologie et toxicologie cliniques, génétique médicale, médecine nucléaire, pathologie, médecine pharmaceutique, prévention et santé publique, radiologie, radio-oncologie/radiothérapie, médecine légale, médecine tropicale et des voyages.

\section{Grandes régions}

Arc lémanique: GE, VD, VS

Espace Mittelland: BE, FR, JU, NE, SO

Suisse du Nord-Ouest: AG, BL, BS

Zurich: ZH

Suisse orientale: AI, AR, GL, GR, SG, SH, TG

Suisse centrale: LU, NW, OW, SZ, UR, ZG

Tessin: TI

\section{Sujets d'actualité du forum \\ 80}

Venez débattre avec nous! Dans la rubrique forum, nous présentons régulièrement des sujets d'actualité politique, économique et scientifique ayant trait au système de santé. Donnez votre avis ou commentez les affirmations de vos confrères. Pour accéder au forum: www.bullmed.ch/forum/ 